\title{
A CONSIDERATION FOR THE LIGHT ENVIRONMENTAL MODELING UNDER TROPICAL RAINFOREST CANOPIES
}

\author{
M. Yoshimura a, ${ }^{\text {a }}$. Yamashita ${ }^{\mathrm{c}}$ \\ ${ }^{a}$ PASCO Corporation, PASCO Research Institute, 2-8-10 Higashiyama, Meguro-ku, Tokyo, Japan -gr4m-ysmr@asahi-net.or.jp \\ b Tokyo University of Agriculture and Technology, Faculty of Agriculture and Women's Future Developing Organization, 3-5-8 \\ Saiwai-cho, Fuchu, Tokyo, Japan-meguyama@cc.tuat.ac.jp
}

Commission VII, WG VII/1

KEY WORDS: Light environment, representativeness, PAR, fluctuating, sky condition, characterization

\begin{abstract}
:
Photosynthetic Active Radiation (PAR) is the most important light source for plant photosynthesis. It is known that most of PAR from solar radiation is well absorbed by the surface. The canopy is the surface in forest region, consists an aboveground portion of plant community and formed by plant crowns. On the other hand, incident solar radiation is fluctuating at all times because of fluctuating sky conditions. Therefore, qualitative light environmental measurements in forest are recommended to execute under stable cloudy condition. In fact, it is quite a few opportunities to do under this sky condition. It means that the diffuse light condition without the direct light is only suitable for this measurement.

In this study, we challenged the characterization the forest light environment as its representativeness under no consideration of sky conditions through analysis huge quantities of instantaneous data which obtained under the different sky conditions. All examined data were obtained under the different sky conditions at the tropical rainforest canopy as one of the typical fluctuating sky conditions regions. An incident PAR is transmitted and scattered by different forest layers at different heights. Various PAR data were measured with quantum units as Photosynthetic Photon Flux Density (PPFD) at different forest heights by the quantum sensors. By comparing PPFDs at different heights with an incident PPFD, relative PPFDs were calculated, which indicate the degree of PPFD decrease from the canopy top to lower levels. As the results of these considerations, daily averaging is confirmed to be cancelled sky fluctuating influences.
\end{abstract}

\section{INTRODUCTION}

Remote sensing is only one powerful tool to expand the plant physiological model-based approach spatial-dimensionally. However it is still remained many technical and theoretical issues to be solved. Photosynthetic Active Radiation (PAR), which is solar radiation in the wavelength range from 400 to $700 \mathrm{~nm}$, is the most important for plant photosynthesis at different forest height (Kenzo et al., 2006). It is known that most of PAR is well absorbed by the surface and only a little is reflected. In biology, this surface is the canopy. The canopy is an aboveground portion of plant community and formed by plant crowns. On the other hand, incident solar radiation is fluctuating at all times because of fluctuating sky conditions (Yamashita and Yoshimura, 2009). Therefore, the light environmental measurements in forest have been recommended to execute under stable cloudy condition. In fact, it is quite a few opportunities to do under cloudy condition only, especially in tropical rainforest climate zone. It makes difficult to understand the representativeness of the light environment in tropical rainforest (Yoshimura and Yamashita, 2012).

In this study, we focus on the influence of fluctuating sky conditions to the diurnal and daily changes in the forest light environment, and we measure the incident PAR on the top of canopy and the transmissive PAR with quantum units as Photosynthetic Photon Flux Density (PPFD) at different forest height under tropical rainforest canopy on diverse sky conditions. The relationships between relative PPFD and forest height are examined with averaging in a short time period and one-day. Then we consider the time representativeness of the forest light environment under different weather conditions.

\section{SITE}

Our research site is the tropical rainforest in Lambir hills national park and the western part of Sarawak state, Borneo Island, Malaysia (fig.1). This region is known as the typical tropical weather region without seasonal change. The tropical rainforest also is known as the lug of the earth or the treasury of biodiversity.

The canopy access crane had constructed in March 2000. This crane is about $80 \mathrm{~m}$ tall height with $75 \mathrm{~m}$ arm length (Nakashizuka et al., 2003). Using the gondola of this crane, it can make us possible to access to any point three-dimensionally.

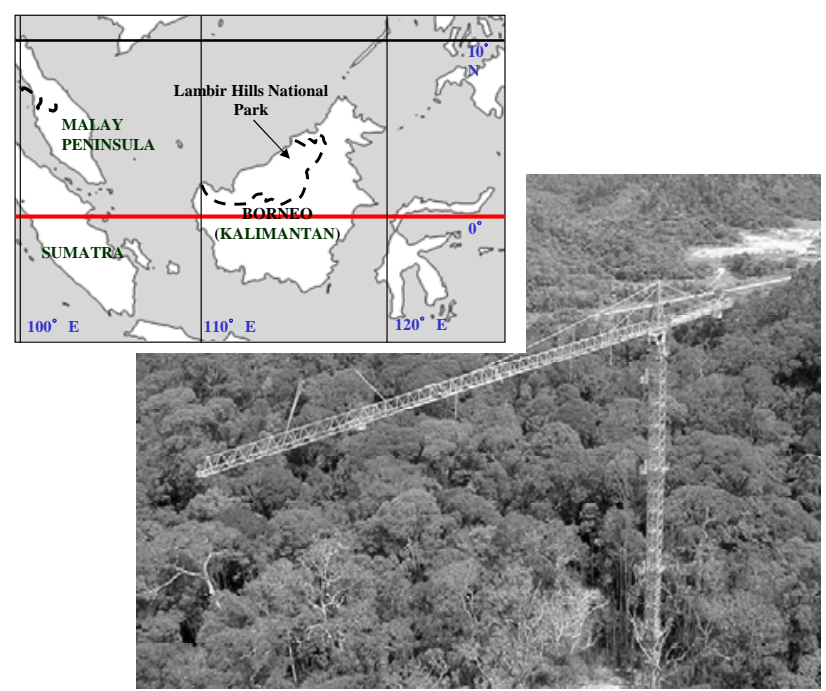

Fig.1 Study site and the canopy access crane 


\section{LIGHT DISTRIBUTION IN FOREST}

Incident solar radiation proceeds from the top of the canopy to the forest floor, and consists of three components. The first is direct radiation, which penetrates the top of the canopy into the forest. The second is diffuse radiation, which is reflected from leaf or ground surfaces. The third is transmissive radiation, which penetrates leaves.

Attenuation of solar radiation is the sum of the three components. Radiation which penetrates the forest is modelled using the Lambert-Beer law, which is expressed in eq.1. According to the Lambert-Beer law, light intensity decays exponentially with Leaf Area Index (LAI). Solar radiation at any height in the forest, $I$, is defined by eq. 1 from the incident solar radiation at the top of the canopy, $I_{0}$, and the corresponding LAI at that height. This equation applies to any plant species. Photosynthetic Photon Flux Density (PPFD, quantum units: $\mu \mathrm{mol} / \mathrm{m}^{2} / \mathrm{s}$ ) is used normally to represent radiation in this kind of analysis. The PPFD is also expressed by PAR. Most incident PAR penetrates leaves and is absorbed effectively by photosynthesizing pigments such as chlorophyll.

$$
I / I_{0}=\exp (-k(\mathrm{LAI}))
$$

where $I_{0}$ is the incident PAR at the top of canopy, $I$ is transmissive PAR and $\mathrm{k}$ is the coefficient of light absorption, and where:

$$
\text { relative } \mathrm{PAR}=I / I_{0}
$$

Light attenuation at any forest height is expressed by the relative PPFD, which is shown in eq.2. This is the ratio between incident and transmissive PPFD, and is calculated from PPFD at a particular height compared with incident PPFD at the top of the canopy (Lowman and Nadkarni 1995). The absorption coefficient $\mathrm{k}$ expresses light absorption below the forest surface.

\section{MEASUREMENTS}

The concept of our measurements for the light environment under tropical rainforest canopy is shown in fig.2. The incident and the transmissive PPFDs measurements are explained as follows.

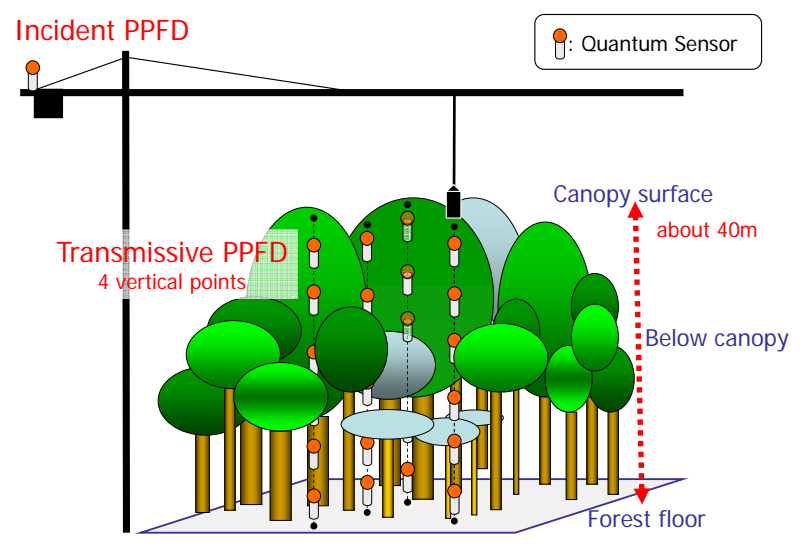

Fig.2 Concept of the forest light environmental measurements

\subsection{Incident PPFD}

The incident PPFD is observed for the standard for relative PPFD calculation. To determine the standard of relative PPFD calculation, a quantum sensor with ultra-small memory (MDS-
MkVL by Alec) was used. The quantum sensor was set up on the top of the observation crane, unobscured by obstacles.

To monitor sky conditions and account for cloud effects on the forest light environment during qualitative forest characterization, whole-sky images were captured with a fisheye lens camera installed at the top of canopy.

\subsection{Transmissive PPFD}

Incident PPFD is transmitted and scattered by different forest layers at different heights. Various PAR data were measured with quantum units at different forest heights by the same quantum sensors. By comparing PPFDs at different heights with the standard PPFD, relative PPFDs are calculated which indicated the degree of PPFD decrease from the canopy top to lower levels.

Fig. 3 shows the target canopy view and the quantum sensors' horizontal and vertical distributions. The upper left figure shows the incident PPFD measurement instruments' view at the top of the crane. The central upper figure shows the distribution of four horizontal measurement points on our objective canopy. At each point 1 to 4, 6 quantum sensors were placed at different heights below the canopy; the upper right image is one of the sensors' views. The lower image depicts six vertical sensors' distributions at four horizontal measurement points.

The S-25 sensor measured the standard PPFD at the top of the canopy for calculation of relative PPFD.

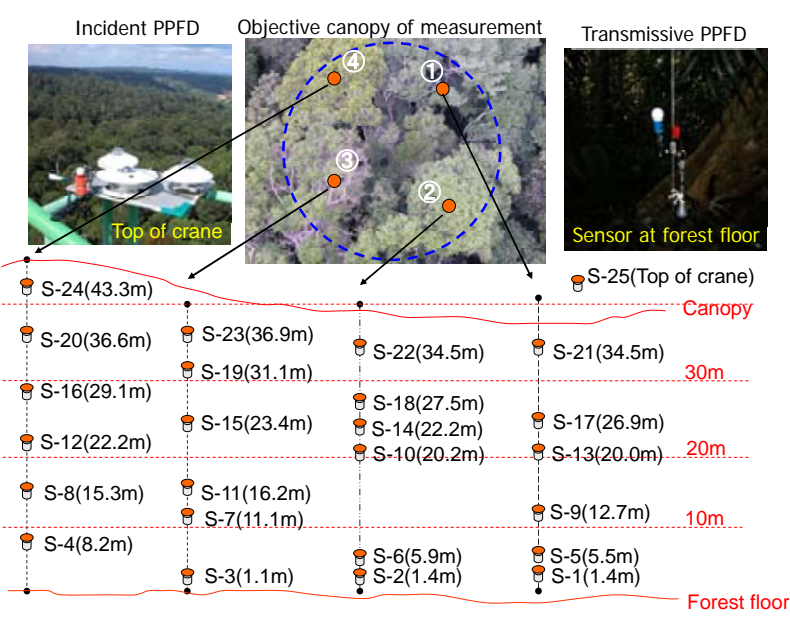

Fig.3 Distribution of target canopy and quantum sensors.

\section{RESULTS}

\subsection{Time representative Consideration}

We observed the light environment under the tropical rainforest canopies for 10 days from $29^{\text {th }}$ August to $7^{\text {th }}$ September in 2005. The weather conditions were mixed by sunny and cloudy.

Here, we considered the time representativeness of light environment under the canopies in our site. Fig. 4 shows the relative PPFD at 24 measurement points with one-day average and 10-days average. Comparing with one-day and 10-days averages, there is no big difference. Three exponential approximations in fig. 4 are derived from the relative PPFD of $1^{\text {st }}$ Sep under cloudy condition, $5^{\text {th }}$ Sep under sunny condition, and 10-days under all weather conditions. There is also no difference between sunny and cloudy days. In this way, it is able to show the time representativeness of light environment in the forest by averaging the one-day relative PPFD regardless of the cloudy and sunny. Also, to derive the approximations as 
exponential from the relations with relative PPFD with forest height, that is, there is a linear relationship between forest height and LAI in our study forest site.

\subsection{Time representativeness on weather Conditions}

The incident and transmissive PARs under sunny condition are easy changeable in short time period by clouds movement. Therefore, we examined the minimum time requirement for the time representativeness of the light environment under different weather conditions.

Fig.5 and 6 show the 30-minute average of relative PPFD with forest height at each measurement point observed on $29^{\text {th }}$ Aug. under sunny condition and $1^{\text {st }}$ Sep. under cloudy condition respectively. These exponentials are derived as approximations from 30-minute averages at 24 measurement points. The variation of relative PPFD at each measurement point and each 30 -minute interval is very large especially more than $20 \mathrm{~m}$ of the sensors' height on sunny day (fig.5). On the other hand, we cannot see the big differences between these measurement points and between 30-minute intervals on cloudy day (fig.6). From these results, we can recognize that the 30 -minute average of relative PPFD measured under cloudy condition has the adequate time representativeness of the light environment in forest.

\section{CONCLUSION}

To characterize the forest light environment as its representativeness under no consideration of weather conditions, we analysed huge quantities of instantaneous data which obtained at the different measurement heights and under weather conditions in the tropical rainforest.

Through the considerations related with the time representativeness and weather conditions in the forest light environment, we could understand the time representativeness of light environment in the forest by averaging the one-day relative PPFD regardless of the cloudy and sunny. Furthermore, we could recognize that the 30-minute average of relative PPFD measured under cloudy condition has the adequate time representativeness as minimum time requirement.

As the future works, we will develop the light environmental modeling under the rainforest canopies by considering the relationship between the forest height and LAI.

\section{REFERENCES}

Kenzo, T., Ichie, T., Watanabe,Y.,Yoneda,R.,Ninomiya, I. and Koike, T., 2006, Change in photosynthesis and leaf characteristics with tree height in five dipterocarpaceae species in a tropical rain forest. Tree Physiology, 26, pp. 865-873.

Lowman, D.M. and Nadkarni, M.N. (Eds.), 1995, Forest Canopies (San Diego, CA: Academic Press).

Nakashizuka, T., Sakai, S. and CHONG, L., 2003, Lambir Hills National Park canopy crane, Malaysia. In Studying Forest Canopies from Above: the International Canopy Crane Network, Y. Basset, V. Horlyck and S.J. Wright (Eds.), pp. 120-125 (Panama: Smithsonian Tropical Research Institute and UNEP).

Yamashita, M. and Yoshimura, M., 2009, The Diurnal Global and Diffuse PAR Change Analysis by Sky Conditions and its Characteristics. Journal of Environmental Information Science, Vol. 37, No.5, pp.7-14.

Yoshimura, M. and Yamashita, M., 2012, Measurement of tropical rainforest three-dimensional light environment and its diurnal change, International Journal of Remote Sensing, 33:3, 848-859.introduction

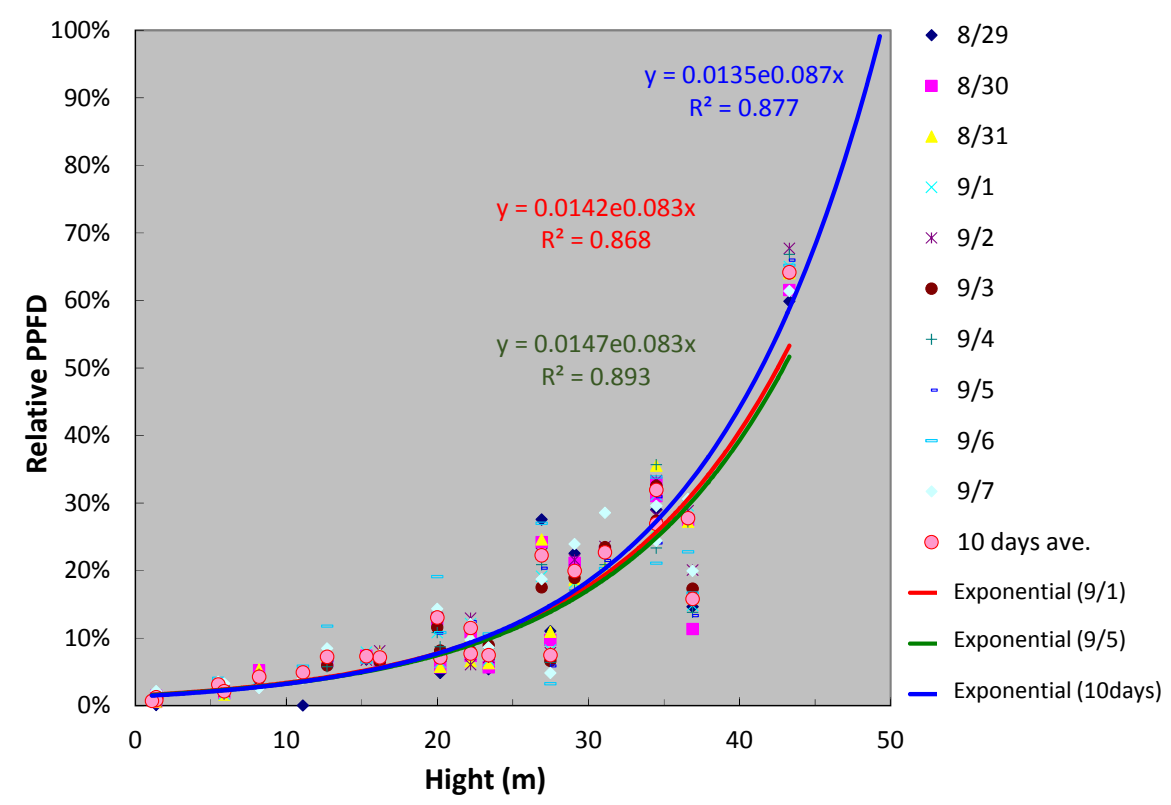

Fig.4 Relations of the relative PPFD and forest height at each measurement points with one-day average and 10-days average. 


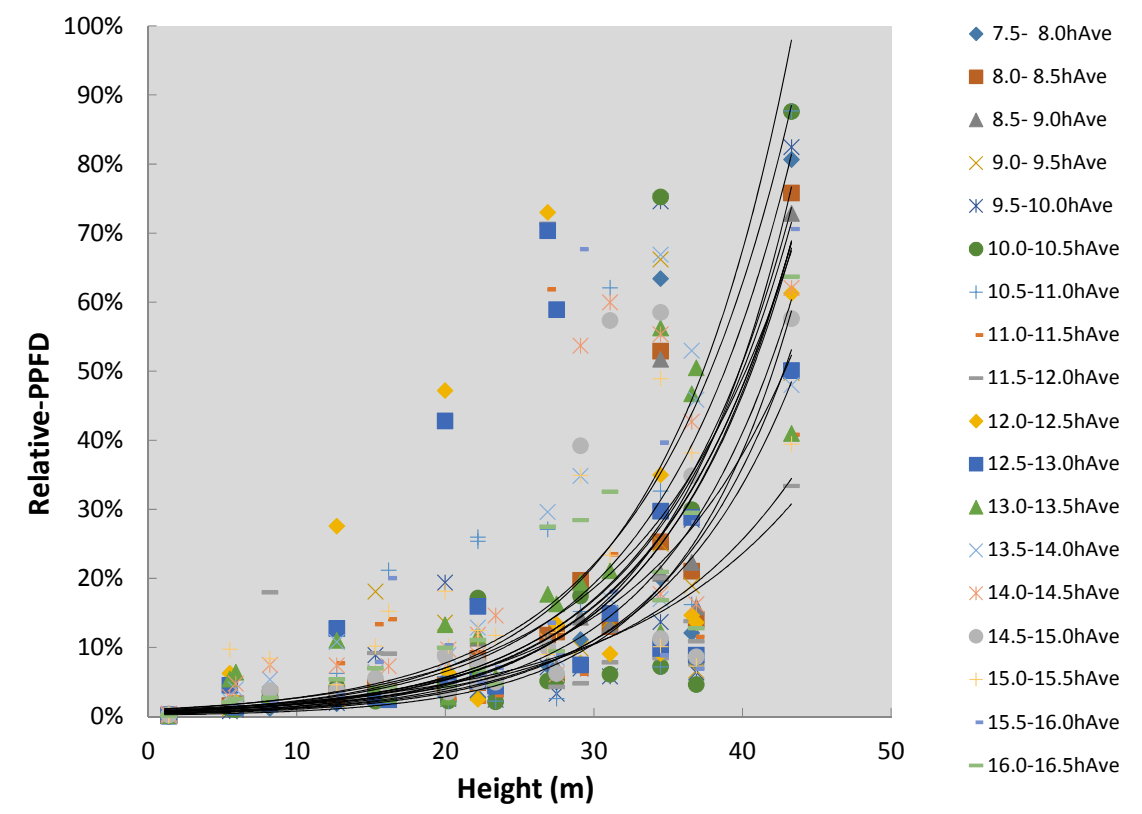

Fig.5 30-minute average of relative PPFD with forest height at each measurement point observed on $29^{\text {th }}$ Aug. under sunny conditions.
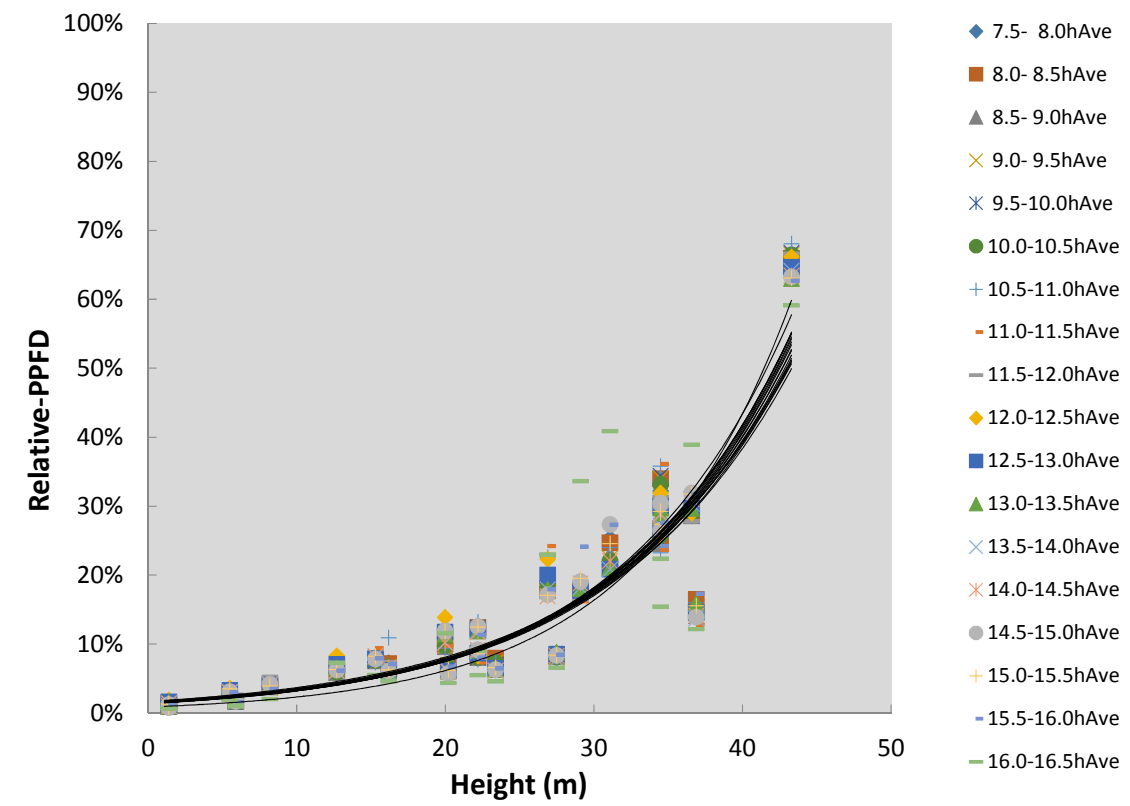

Fig.6 30-minute average of relative PPFD with forest height at each measurement point observed on $1^{\text {st }}$ Sep. under cloudy condition. 